\title{
New Class of Compact Stars at High Density*
}

\author{
E. S. Fraga ${ }^{a \dagger}$ R. D. Pisarski ${ }^{a}$ J. Schaffner-Bielich ${ }^{\mathrm{b}}$ \\ aDepartment of Physics, Brookhaven National Laboratory, \\ Upton, NY 11973-5000, USA \\ ${ }^{\mathrm{b}}$ RIKEN BNL Research Center, Brookhaven National Laboratory, Upton, NY \\ 11973-5000, USA
}

We discuss the equation of state for cold, dense quark matter in perturbation theory, and how it might match onto that of hadronic matter. Certain choices of the renormalization scale correspond to a strongly first order chiral transition, and may generate a new class of small and very dense quark stars. The results for the mass-radius relation are compatible with the recent determination of the mass and the radius of an isolated neutron star by Pons et al.

The investigation of the structure of the phase diagram for strongly interacting matter is a fascinating subject. Moreover, the study of regions of the diagram associated with extreme conditions in temperature and density can reveal new phenomena, and provide a better understanding of Quantum Chromodynamics (QCD). Nevertheless, in spite of the increasing interest this field has attracted during the last decade, the mapping of the QCD phase diagram is still in its infancy, and most of its regions remain mystifying and challenging. An exception is given by the region of nonzero temperature and very small densities. There, guidance is given by lattice simulations [1] and experiments in heavy ion collisions [2]. On the other hand, an increasing amount of precise astrophysical data provides a new resource to probe QCD at large density. The natural candidates to study are neutron stars, whose interior might be dense enough to allow for the presence of chirally symmetric quark matter [3 8]. Concretely, certain combinations of observables, such as the mass-radius relation, may be useful to constrain the equation of state for strongly interacting matter.

Neutron star models predict a maximum mass in the range $\approx 1.4-2.0 M_{\odot}$, with a radius $\approx 10-15 \mathrm{~km}$. The values for the maximum mass are consistent with the pulsar data [3]. The conventional theoretical approach used to describe a hybrid star, a neutron star with quark matter in the core, or a strange star is the bag model, with at best a correction $\sim \alpha_{s}$ from perturbative QCD [6]. If the bag constant is fit from hadronic phenomenology, then the gross features of those stars are very similar to those expected for neutron stars, especially in the region of mass compatible with the pulsar observations. Through the

\footnotetext{
${ }^{*}$ Presented at the International Conference on Statistical QCD, Bielefeld, Germany, 26-30 Aug 2001.

${ }^{\dagger}$ Present address: LPT, Université Paris-Sud XI, Bâtiment 210, F-91405 Orsay, France.
} 
years, the study of compact hybrid stars has produced a myriad of different models, with all sorts of possible layers and condensates, but with numbers very similar to the ones presented above [3]. Nevertheless, recent astrophysical results for the mass-radius diagram seem to point in a direction unforeseen by traditional models [9].

In [10] we considered cold, dense quark matter in perturbation theory to $\sim \alpha_{s}^{2}$ 《, analyzing its implications for the physics of quark stars. We used perturbation theory not because it is a good approximation, but simply to model the possible nonideality in the equation of state of cold, dense QCD. Here we extend this discussion, and consider as well how to match this "perturbative" equation of state onto that of hadrons. We emphasize especially the comparison between the pressure of the hadronic and quark phases. While our results are admittedly similar to other theoretical approaches [11], we try to place these results in a more general context, in terms of the QCD phase diagram. Lastly, most intriguing is the recent determination of the mass and the radius of an isolated neutron star, which suggests an "implausible" equation of state [9].

Assume that the chiral phase transition occurs at a chemical potential $\mu_{\chi}$. Our perturbative equation of state applies in the chirally symmetric phase, when the quark chemical potential $\mu>\mu_{\chi}$. In this phase, the effects of a strange quark mass, $m_{s} \approx 100 \mathrm{MeV}$ [12], are small relative to the quark chemical potentials, $\mu>300 \mathrm{MeV}$. Thus we take three flavors of massless quarks with equal chemical potentials [3, 13]. How the equation of state for quarks matches onto that of hadrons is discussed later.

The thermodynamic potential of a plasma of massless quarks and gluons was calculated perturbatively to $\sim \alpha_{s}^{2}$ by Freedman and McLerran [4] and by Baluni [5], using the momentum-space subtraction (MOM) scheme. The MOM coupling is related to that in the modified minimal subtraction scheme, $\overline{\mathrm{MS}}$, as [10,14]

$\frac{\alpha_{s}^{\mathrm{MOM}}}{\pi}=\frac{\alpha_{s}^{\overline{\mathrm{MS}}}}{\pi}\left[1+\mathcal{A} \frac{\alpha_{s}^{\overline{\mathrm{MS}}}}{\pi}\right] ;$

$\alpha_{s}=g^{2} /(4 \pi)$, with $g$ the QCD coupling constant, and $\mathcal{A}=151 / 48-(5 / 18) N_{f}$, with $N_{f}$ the number of massless flavors. In the $\overline{\mathrm{MS}}$ scheme, to $\sim \alpha_{s}^{2}$ the thermodynamic potential is 10

$\Omega(\mu)=-\frac{N_{f} \mu^{4}}{4 \pi^{2}}\left\{1-2\left(\frac{\alpha_{s}}{\pi}\right)-\left[G+N_{f} \ln \frac{\alpha_{s}}{\pi}+\left(11-\frac{2}{3} N_{f}\right) \ln \frac{\bar{\Lambda}}{\mu}\right]\left(\frac{\alpha_{s}}{\pi}\right)^{2}\right\}$,

where $G=G_{0}-0.536 N_{f}+N_{f} \ln N_{f}, G_{0}=10.374 \pm .13$, and $\bar{\Lambda}$ is the renormalization subtraction point. We take the scale dependence of the strong coupling constant, $\alpha_{s} \equiv$ $\alpha_{s}(\bar{\Lambda})$ to three loop order [15]. In $\overline{\mathrm{MS}}$ scheme, the thermodynamic potential is manifestly gauge invariant. However, there remains a dependence on $\bar{\Lambda} / \mu$ that, in principle, can be freely chosen. Although the results are significantly sensitive to the particular value of this free parameter, this choice is tightly constrained by the physics. (It is not especially sensitive to what order in the loop expansion one used for the running of the coupling constant, or for the pressure.)

With this "perturbative" equation of state — used far outside the region of validity for perturbation theory - reasonable values turn out to be in the range $2 \leq \bar{\Lambda} / \mu \leq 3$. This comes about from the most elementary considerations of how to match the quark and hadronic equations of state. 


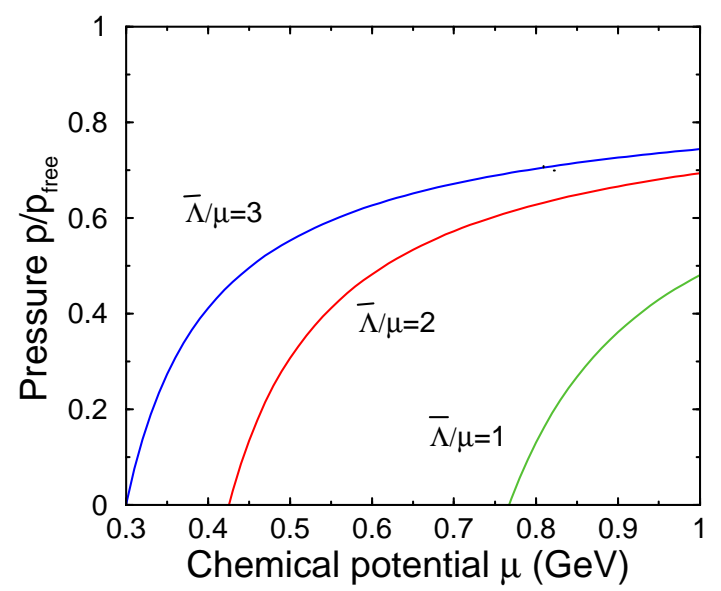

Figure 1. The total pressure up to order $\sim \alpha_{s}^{2}$, relative to the pressure of an ideal gas, $p_{\text {free }}$, as a function of $\mu$ for different choices of $\bar{\Lambda} / \mu$.

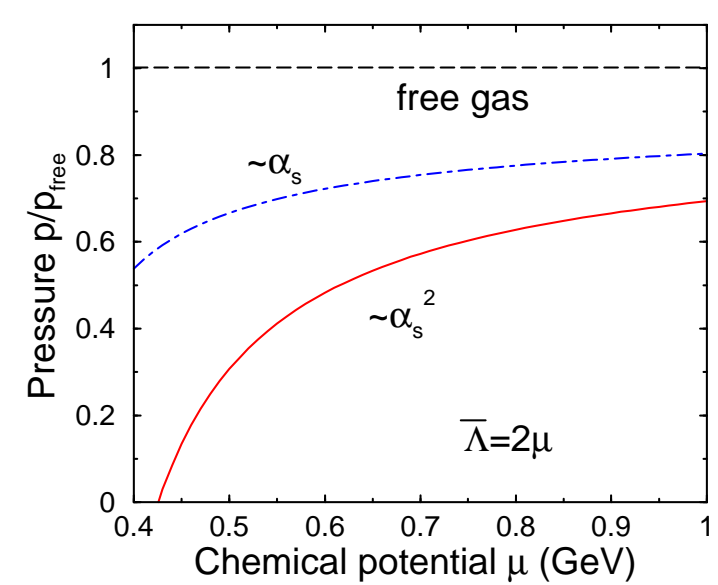

Figure 2. The total pressure, relative to the pressure of an ideal gas, $p_{\text {free}}$; including terms to order $\sim \alpha_{s}$ and to order $\sim \alpha_{s}^{2}$, as a function of $\mu ; \bar{\Lambda}=2 \mu$.

In Fig. 11 we show the pressure for some illustrative choices of $\bar{\Lambda} / \mu=1,2,3$. In each case, the pressure vanishes at some $\mu=\mu_{c}$. As the chemical potential of any field must be greater than the mass, and as the chemical potential of quarks is one third that of baryons, the hadronic pressure vanishes at $\mu_{\min }=939 / 3=313 \mathrm{MeV}$ (This neglects the small effects of nuclear binding, which contribute at most $\sim 15 / 3=5 \mathrm{MeV})$. Then $\mu_{c}$ must be greater than $\mu_{\text {min }}$; from Fig. [1, this requires that $\bar{\Lambda}$ is less than $3 \mu$. Conversely, $\mu_{c}$ cannot be too large. Dense hadrons exert pressure, so before the quark pressure goes negative, a transition to massive quarks and hadronic matter occurs. For example, when $\bar{\Lambda}=\mu$, one can show that hadronic matter must persist to very high densities, maybe thirty times nuclear matter density. This is unlikely; indeed, below we see that the worry is not how small the hadronic pressure is, but how big.

Thus, reasonable values of $\bar{\Lambda}$ are in the range of $\mu \rightarrow 3 \mu$. Choosing the value $\bar{\Lambda}=2 \mu$, we can get an idea of how well perturbation theory works for large chemical potential. At $\mu=1 \mathrm{GeV}$, where $\alpha_{s}=0.31$, the perturbative corrections to ideality are $\sim 30 \%$; at $\mu=100 \mathrm{GeV}$, where $\alpha_{s}=0.095$, they are still $7 \%$. Even so, as we argued in Ref. [10, the perturbative series at $T=0$ and $\mu \neq 0$ may be much better behaved than at $T \neq 0$ and $\mu=0$ [16]. While for $T \neq 0, \mu=0$ we have an expansion in $\sqrt{\alpha_{s}}$, for $T=0, \mu \neq 0$ it is a perturbative series in $\alpha_{s}$ and $\alpha_{s} \log \left(\alpha_{s}\right)$. Besides, at nonzero density (and $T=0$ ), the $\sim \alpha_{s}$, and $\sim \alpha_{s}^{2}$ corrections both act to lower the pressure. It is encouraging that the $\sim \alpha_{s}^{2}$ corrections are smaller than those at $\sim \alpha_{s}$ down to $\mu=0.54 \mathrm{GeV}$. In contrast, at nonzero temperature (and $\mu=0$ ), terms oscillate in sign, and the naive perturbative series is not well behaved until extremely high temperatures, $\sim 10^{7} \mathrm{GeV}[17]$.

Of course a much better estimate of the quark pressure could be obtained if the terms $\sim \alpha_{s}^{3}\left(\log ^{2}\left(\alpha_{s}\right), \log \left(\alpha_{s}\right), 1\right)$ were computed. Unlike nonzero temperature - where only the $\sim \alpha_{s}^{3}\left(\log ^{2}\left(\alpha_{s}\right), \log \left(\alpha_{s}\right)\right)$ are sensible to compute, for dense QCD all terms in the 
perturbative expansion of the thermodynamic potential are well defined.

This does not imply that a given value of $\alpha_{s}$, which is adequate to compute the thermodynamic potential, works equally well for all other quantities. In particular, the gaps for color superconductivity are nonperturbative, $\phi \sim \exp \left(-1 / \sqrt{\alpha_{s}}\right)$ [18, 19], and much smaller values of $\alpha_{s}$ appear to be required to reliably compute them [20]. Perturbative results, unjustly extrapolated down to $\mu \sim 400 \mathrm{MeV}$, suggest that these gaps are $\sim 30 \mathrm{MeV}$ [21]. As the relative change in the thermodynamic potential is only $\sim(\phi / \mu)^{2}$, then, for the equation of state in QCD, color superconductivity is a negligible effect.

The structure of a quark star is determined by the solution to the Tolman-OppenheimerVolkov (TOV) equations [3]. Given the equation of state $p=p(\epsilon)$, one can integrate the TOV equations from the origin until the pressure vanishes at the edge of the star, obtaining a characteristic curve in the mass-radius diagram. In matching the pressure of quarks onto that of hadrons, there are two possible scenarios. In the first, the pressure of hadrons rise quickly, smoothly matching onto that of quarks. Since the pressure of the hadronic and quark phases are similar, so are the densities (as the slopes of the pressure); then the chiral phase transition is either weakly first order, or just crossover. In the second case, the hadronic pressure is assumed to always remain small. This gives a strongly first order chiral transition, as one goes abruptly from a phase of dilute hadrons to one of dense quarks [22]. A sketch of both cases is depicted in Fig. 3, where the equation of state for massless quarks is taken to be the one with $\bar{\Lambda}=2 \mu$.

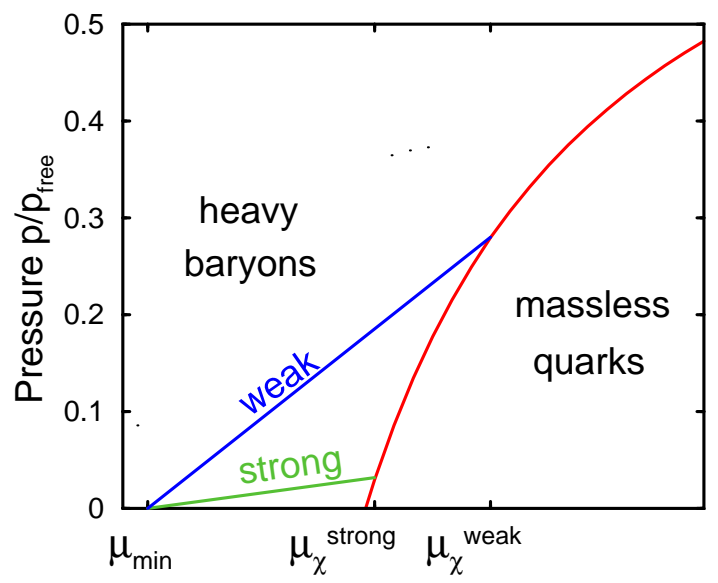

Figure 3. Sketch of the total pressure, relative to the pressure of an ideal gas, $p_{\text {free}}$, for a weak (upper line) and a strong (lower line) chiral transition as discussed in the text.

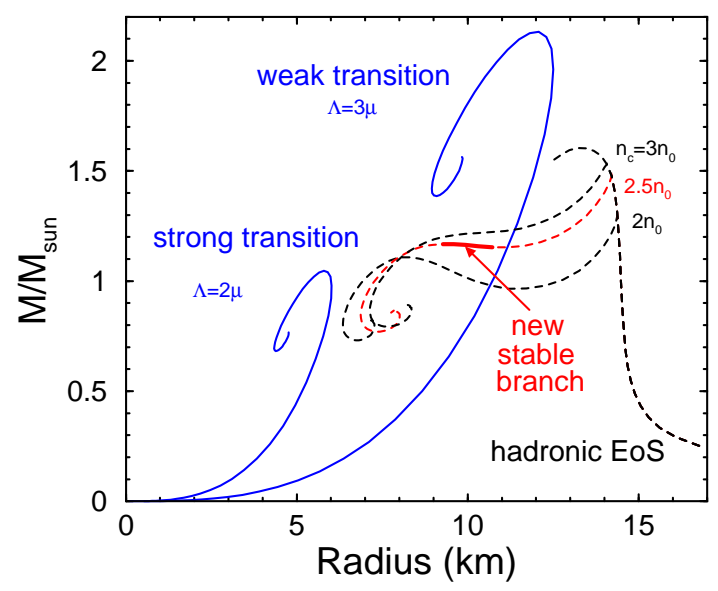

Figure 4. Mass-radius relation for pure quark stars (solid lines) and hybrid stars (dashed lines) for different critical densities for the chiral transition.

Figure 1 shows the mass-radius diagram for these two cases. A weakly first order chiral transition is illustrated by the choice of $\bar{\Lambda}=3 \mu$. The quark pressure vanishes at $\mu_{c}=\mu_{\text {min }}$, so that the pressure of dense hadrons grows immediately, and becomes equivalent to the 
pressure of dense quarks. The numerical solution of the TOV equations for such an equation of state generates hybrid stars with maximum masses and radii compatible with those from conventional neutron star models, $M \sim 2 M_{\odot}$ and $R \sim 12 \mathrm{~km}$.

For a strongly first order chiral transition, as with $\bar{\Lambda}=2 \mu$, there is a branch along which stars are predominantly composed of dilute hadrons, with small pressure. These look like ordinary neutron stars. In addition, there is a new branch in the mass-radius diagram. These are predominantly composed of very dense quarks with a thin hadronic mantle. The pressure is like that of QCD with a larger bag constant $\left(\mu_{\min }\right.$ is larger). Working out how the maximum mass and radius scale with an (effective) bag constant, one finds maximum masses $M \sim 1 M_{\odot}$ and radii $R \sim 6 \mathrm{~km}$. The new branch was suggested previously by several groups $\llbracket 7,8]$.

Since we have no plausible hadronic equation of state with small pressure, we simply ignore it, and take the quark equation of state down to the point where it vanishes. In comparison, in Figure 1 we also show the mass-radius relation for a hadronic equation of state (a mean-field equation of state with parameter set TM1, including hyperons [23]). Matching the hadronic equation of state onto the one for the quark phase is done by fixing a critical density. For critical densities about twice that of nuclear matter, we find a new branch appears, characterized by a second bump in the mass-radius curve.

Experimentally, Pons et al [9] find that for an isolated, nearby neutron star, the masses and radii are exactly what one expects for a new branch of quark stars, with $M \approx .9 M_{\odot}$ and $R \approx 6 \mathrm{~km}$.

The difficulty is really in understanding how hadrons can have a small pressure [24]. Akmal, Pandharipande, and Ravenhall [25] have found that to a very good approximation, in pure neutron matter the energy per baryon (minus its rest mass) is approximately linear in the baryon density, $n$ :

$\frac{E}{A}-m=\frac{\epsilon}{n}-m=15 \mathrm{MeV}\left(\frac{n}{n_{0}}\right)$.

where $\epsilon$ is the energy density and $n_{0} \sim .16$ baryons $/ \mathrm{fm}^{3}$ is the saturation density for nuclear matter. These authors used a wide variety of different models for the nucleonnucleon interaction, and find that up to $n \approx n_{0}$, all models are in close agreement. Even at such "low" densities, this behavior itself is already signs of a highly nonideal Fermi liquid, since free fermions give $\epsilon / n-m \sim n^{2 / 3}$, not $\sim n$. By two times nuclear matter density, the discrepancies between different models is already $\sim 50 \%$, and grow sharply with density.

The energy on the right hand side of (3), $\sim 15 \mathrm{MeV}$, is very small on hadronic scales. It is much smaller than the pion decay constant, which one might expect is the natural scale. What is remarkable about the results of Akmal et al. [25] is that the energies are small not only for nuclear matter, which has a nonzero binding energy, but even for pure neutron matter, which is unbound. This suggests to us that the smallness of the right hand side of eq. (3) is a generic property of baryons interacting with pions, etc., and is not due to any special tuning. Using this result, however, we can reliably compute the pressure of pure neutron matter at nuclear matter density:

$p_{\text {hadron }}=n^{2} \frac{\partial}{\partial n}\left(\frac{\epsilon}{n}\right)=15 \mathrm{MeV} \frac{n^{2}}{n_{0}}=.04 p_{\text {free }}\left(\frac{n}{n_{0}}\right)^{2}$ 
Here $p_{\text {free }}$ is the pressure for three flavors of massless quarks; for the purposes of discussion, in $p_{\text {free }}$ we have taken $\mu=313 \mathrm{MeV}$. Thus at nuclear matter density, the pressure is $4 \%$ that for ideal quarks, which is comfortably small. For the hadronic pressure to remain small relative to the quark pressure, the transition must occur at remarkably low densities, maybe twice that of nuclear matter.

We do not have a good reason for why the hadronic pressure should remain small to the chiral transition. But since we don't understand why it is small in nuclear matter, we feel free to extrapolate; maybe the hadronic pressure is suppressed in the limit of a large number of colors. Such "implausible" equations of state, with a small hadronic pressure, can be constructed mathematically [26].

If small and dense quark stars occur, then their production is probably different from those of ordinary neutron stars. Models of supernova collapses suggest that the mass of a proto-neutron star formed by the collapse of the iron core is in excess of $1 M_{\odot}$ as the initial mass of the iron core is well above $1 M_{\odot}$. Neutron stars can not collapse to quark stars, as they have to expel most of their mass to achieve a stable quark star. White dwarfs do not collapse to more compact objects in type Ia supernova as the explosion destroys the whole star. In the early universe, blobs of strange quark matter formed during the quark-hadron phase transition can not be larger than $10^{-9} M_{\odot}$ due to the horizon at $t=10^{-6}$ s after the big-bang [27].

Nevertheless, there might be some exotic ways to produce quark stars. If white dwarfs can develop an iron core by some (so far not established) reason, the collapse of this core can form a low-mass quark star. Alternately, if inflation occurs at the quark-hadron phase transition, then larger strange quark stars, with $M=0.01-10 M_{\odot}$, can be formed [28].

The existence of a new class of small and dense compact stars is an exciting possibility. While superconducting condensates do not matter for the pressure, they do dominate their electromagnetic response. Whatever the magnitude of the gaps for color superconductivity, versus those for hadrons, there should be a multitude of ways in which stars composed of color-flavor locked quarks differ from those of superconducting (and superfluid) baryons. After all, in this scenario the stars are never really hybrids, but either mainly hadronic, or mainly quark.

We thank G. Baym, J. Beacom, H.-T. Janka, A. Olinto, A. Peshier, K. Rajagopal, D. Rischke, and especially L. McLerran for fruitful discussions. We thank the U.S. Department of Energy for their support under Contract No. DE-AC02-98CH10886; E.S.F., for the support of CNPq (Brazil); and J.S.B., for the support of RIKEN and BNL.

\section{REFERENCES}

1. F. Karsch, arXiv:hep-ph/0103314.

2. Proceedings of Quark Matter 2001, to appear in Nucl. Phys. A; http://rhic.bnl.gov/qm2001].

3. N. K. Glendenning, Compact Stars - Nuclear Physics, Particle Physics, and General Relativity (Springer, New York, 2000); H. Heiselberg and M. Hjorth-Jensen, Phys. Rept. 328, 237 (2000).

4. B. A. Freedman and L. D. McLerran, Phys. Rev. D16, 1130 (1977); ibid., D16, 1147 (1977); ibid., D16, 1169 (1977); ibid., D17, 1109 (1978). 
5. V. Baluni, Phys. Rev. D 17, 2092 (1978).

6. A. R. Bodmer, Phys. Rev. D 4, 1601 (1971). E. Witten, Phys. Rev. D 30, 272 (1984). C. Alcock, E. Farhi and A. Olinto, Astrophys. J. 310, 261 (1986); P. Haensel, J. L. Zdunik, and R. Schaeffer, Astron. Astrophys. 160, 121 (1986).

7. U. H. Gerlach, Phys. Rev. 172, 1325 (1968); B. Kämpfer, Jour. Phys. A 14, L471 (1981); Jour. Phys. G 9, 1487 (1983).

8. N. K. Glendenning and C. Kettner, Astron. Astrophys. 353, L9 (2000); K. Schertler, C. Greiner, J. Schaffner-Bielich and M. H. Thoma, Nucl. Phys. A677, 463 (2000).

9. J. A. Pons, F. M. Walter, J. M. Lattimer, M. Prakash, R. Neuhauser and P. An, arXiv:astro-ph/0107404.

10. E. S. Fraga, R. D. Pisarski and J. Schaffner-Bielich, Phys. Rev. D 63, 121702 (R) (2001).

11. A. Peshier, B. Kämpfer and G. Soff, arXiv:hep-ph/0106090. A. Peshier, this volume (2001).

12. T. Blum, A. Soni, and M. Wingate, Phys. Rev. D60, 114507 (1999).

13. K. Rajagopal and F. Wilczek, Phys. Rev. Lett. 86, 3492 (2001) arXiv:hepph/0012039.

14. Due to the unusual MOM scheme used by Freedman and McLerran, the translation to $\overline{\mathrm{MS}}$ is a little tricky. The conversion factor was calculated independently by J. P. Blaizot, E. Iancu and A. Rebhan, Phys. Rev. D 63, 065003 (2001). See also [5].

15. D. E. Groom et al., Eur. Phys. J. C15, 1 (2000).

16. R. D. Pisarski and D. H. Rischke, Phys. Rev. D 61, 051501(R) (2000).

17. E. Braaten, this volume (2001) and references therein.

18. D. T. Son, Phys. Rev. D59, 094019 (1999); R. D. Pisarski and D. H. Rischke, Phys. Rev. Lett. 83, 37 (1999).

19. K. Rajagopal and F. Wilczek, arXiv:hep-ph/0011333.

20. W. E. Brown, J. T. Liu and H. Ren, Phys. Rev. D61, 114012 (2000); ibid. D62, 054013 (2000); ibid. D62, 054016 (2000); K. Rajagopal and E. Shuster, ibid. 62, 085007 (2000).

21. D. H. Rischke, this volume (2001).

22. In the $\mu-T$ plane, if the chiral critical endpoint exists at relatively high temperatures, as suggested by: M. Stephanov, K. Rajagopal and E. Shuryak, Phys. Rev. Lett. 81, 4816 (1998); Phys. Rev. D 60, 114028 (1999), then it is natural that the chiral transition at $\mu \neq 0, T=0$, is strongly first order.

23. J. Schaffner and I. N. Mishustin, Phys. Rev. C 53, 1416 (1996)

24. When $\mu<\mu_{\chi}$, the pressure is small if it is dominated by the thermal Polyakov loop (R. D. Pisarski, Phys. Rev. D 62, 111501 (2000)), and if its expectation value is also small. Why this should be true, especially if $N_{f} \approx N_{c}$, is unclear 29]. Perhaps $m_{s} \neq 0$ has a greater effect when $\mu<\mu_{\chi}$ (kaons are much heavier than pions) than for $\mu>\mu_{\chi}$. 25. A. Akmal, V. R. Pandharipande and D. G. Ravenhall, Phys. Rev. C 58, 1804 (1998). 26. E. S. Fraga, R. D. Pisarski and J. Schaffner-Bielich, work in progress.

27. J. Madsen, arXiv:astro-ph/9809032.

28. N. Borghini, W. N. Cottingham and R. Vinh Mau, J. Phys. G 26, 771 (2000)

29. A. Dumitru and R. D. Pisarski, hep-ph/0010083. 